\title{
Le profilage des demandeurs d'emploi : modèle américain versus modèle néerlandais
}

Profiling for Jobseekers, American model versus Dutch model

\section{Nathalie Georges}

\section{(2) OpenEdition}

\section{Journals}

Édition électronique

URL : http://journals.openedition.org/travailemploi/2156

DOI : 10.4000/travailemploi.2156

ISSN : 1775-416X

Éditeur

DARES - Ministère du Travail

\section{Édition imprimée}

Date de publication : 30 décembre 2007

Pagination : 7-20

ISSN : 0224-4365

\section{Référence électronique}

Nathalie Georges, «Le profilage des demandeurs d'emploi : modèle américain versus modèle néerlandais », Travail et Emploi [En ligne], 112 | octobre-décembre 2007, mis en ligne le 05 novembre 2010, consulté le 01 mai 2019. URL : http://journals.openedition.org/travailemploi/2156 ; DOI 10.4000/travailemploi.2156 


\title{
Le profilage des demandeurs d'emploi: modèle américain versus modèle néerlandais
}

\author{
Nathalie Georges (*)
}

L'article compare deux approches du profilage, l'un, américain qui s'appuie sur une analyse économétrique des caractéristiques des chercheurs d'emplois afin d'identifier les personnes à haut risque; et l'autre, néerlandais, qui tout en utilisant la statistique, s'appuie davantage sur ce que l'auteure appelle la «négociation » entre le chômeur et son conseiller de placement. L'auteure se pose la question de la construction effective de tels outils et des voies qu'elle a empruntées et examine si les expériences connues à ce jour ont atteint leurs objectifs. Le profilage n'est-il pas que la première étape d'une logique de parcours et d'individualisation de l'accompagnement?

Le principe du profiling est né aux États-Unis au début des années 1980, lorsque l'augmentation rapide du chômage de longue durée(1) en a révélé la spécificité, encourageant une approche différant des politiques de l'emploi traditionnelles. Il est apparu en effet que les demandeurs d'emploi qui en étaient victimes présentaient une employabilité particulièrement faible par rapport aux chômeurs de courte durée, en partie expliquée par la récurrence de certaines de leurs caractéristiques individuelles qui semblaient les défavoriser sur le marché du travail. De nombreuses réflexions visant à prévenir l'enfoncement dans le chômage de longue durée, et s'appuyant sur l'hypothèse d'une probabilité de retour à l'emploi inégalement distribuée(2), ont alors conduit à développer l'idée du profilage comme technique de détection précoce des demandeurs d'emploi potentiellement les plus défavorisés.

(*) Centre d'études de l'emploi, nathalie.georges@cee.enpc.fr L'auteur remercie, pour leur relecture attentive et leurs conseils avisés, Guillaume Delautre, Jacques Freyssinet, Bernard Gazier, Marie-Thérèse Letablier et Dominique Méda.

(1) La part des chômeurs de longue durée (plus de six mois de chômage) est passée de $10,7 \%$ en 1980 à $23,9 \%$ en 1983 .

(2) Lors de l'inscription au chômage, selon l'hypothèse d'hétérogénéité inobservée, et au fur et à mesure que la période de chômage se prolonge, selon celle de dépendance d'état. Decreuse et Di Paola (2002) montrent que les deux effets coexistent la plupart du temps. Le premier justifie un ciblage sur les demandeurs d'emploi les plus défavorisés, le second la mise en œuvre de mesures préventives pour limiter le risque d'éloignement du marché du travail. Une troisième explication de l'enfoncement dans le chômage de longue durée est aussi à prendre en compte, celle de la stigmatisation dont peuvent être victimes les chômeurs de longue durée, et qui conduit à leur discrimination par les employeurs. Il est alors intéressant de faire intervenir le profilage de manière précoce.
La construction et à la mise en place effectives d'un outil de calcul d'un risque de chômage de longue durée ont fait suite à la crise économique américaine du début des années 1990. Opérationnel dès 1994, le profilage américain a servi de modèle d'abord aux pays anglo-saxons (Australie 1994, Royaume-Uni 1994, Canada 1994, testé en Nouvelle-Zélande(3)) puis aux pays d'Europe continentale qui se sont progressivement tournés vers ce type d'approche (Pays-Bas 1999, Danemark 2004, Allemagne 2005, France 2006, et d'autres pays où il est en cours d'expérimentation: Belgique, Finlande, Grèce, Hongrie, Italie, Slovaquie, Suède et Suisse), encouragés dans cette voie par la nécessité de mettre en œuvre la Stratégie européenne pour l'emploi.

Deux idéaux-types de profilage se détachent des expériences les plus abouties:

- d'un côté, le modèle américain, qui privilégie les méthodes statistiques, et les considère comme suffisamment fiables pour orienter efficacement les demandeurs d'emploi. Dans ce cas, la décision d'orientation repose sur le seul résultat quantifié du profilage;

- de l'autre, le modèle néerlandais, qui refuse de se fier entièrement au résultat statistique, et le rejette aujourd'hui de plus en plus, insistant sur la place centrale qui doit être conservée à l'entretien avec un conseiller du service public de l'emploi (SPE). Le profilage statistique n'est alors qu'un outil d'aide à la décision, auquel le conseiller peut éventuellement recourir à l'appui de sa propre expertise.

(3) Depuis 1994, plusieurs études recommandent l'utilisation de modèles statistiques de profilage, mais le gouvernement néozélandais leur a préféré une politique incitative fondée sur la sanction ( $c f$. OBBEN, 2001). 
Le présent article a pour objet de caractériser ces deux idéaux-types, dans l'optique de dégager deux modèles concurrents d'utilisation d'un même outil. L'objectif est de comprendre comment et pourquoi deux modèles opposés se sont développés à partir de constats et besoins initiaux comparables, afin d'éclairer le débat qui se tient actuellement en France autour de la mise en place d'un tel outil.

Notre connaissance des systèmes institutionnels des pays étudiés s'appuie essentiellement sur la littérature existante, et sur des informations que nous avons recueillies directement auprès des SPE. Les deux expériences de profilage qui nous servent d'exemple ont été évaluées et nous avons eu accès aux principaux résultats, sur lesquels s'appuient nos conclusions. Après une brève clarification de la définition du profilage, ce travail bibliographique permet de présenter les expériences empiriques qui fondent notre typologie de manière à pouvoir les comparer entre elles, en synthétisant les conclusions de l'observation dans une description idéale-typique des modèles, afin de proposer, pour finir, des hypothèses explicatives des choix différentiels, et de tester la pertinence du rapprochement des différents systèmes de profilage au sein des deux modèles dominants.

\section{Retour sur la définition du profilage}

Si l'on se contente de définir le profilage comme une action préventive et ciblée sur les potentiels chômeurs de longue durée (CLD), il est possible d'avancer que la plupart des pays occidentaux se sont tous déjà dotés d'une méthode de profilage. C'est d'ailleurs la conclusion qu'avaient livrée l'OCDE (2002) et l'étude du cabinet Pls Ramboll Management (2001) à la Commission européenne qui souhaitait promouvoir le profilage. En effet, il est aujourd'hui communément admis que les chances de retour à l'emploi sont inégalement distribuées, et que certains chômeurs ont besoin d'une aide plus intensive que d'autres pour éviter de devenir CLD. Les SPE ont donc depuis longtemps cherché à segmenter les nouveaux inscrits en fonction de leur espérance de retour à l'emploi, afin d'aider chacun en fonction de ses besoins individuels (BENARROSH, 2000).

Cependant, défini comme simple outil de segmentation dans le but de cibler les demandeurs d'emploi les plus en difficulté, le profilage peut englober des pratiques très diverses, et très éloignées de l'exemple fondateur américain (et donc peu comparables, $c f$. encadré 1 ). Il est donc utile de cadrer brièvement ce que nous considérons ici comme un outil de profilage.

\section{Pourquoi profiler?}

Le choix de recourir au profilage se justifie par trois constats principaux qui font consensus parmi les SPE des pays concernés :

- le chômage de longue durée nécessite un traitement spécifique, puisqu'il ne baisse pas mécaniquement avec une amélioration de conjoncture (phénomène d'hystérèse) (OCDE, 1993) et qu'il est intimement corrélé à la situation individuelle des demandeurs d'emploi (FougÈre, 2000). Il convient donc de développer, parallèlement à la politique de l'emploi traditionnelle, des outils adaptés à un accompagnement personnalisé des demandeurs d'emploi, et en particulier des CLD, en suivant une logique de parcours en fonction du profil de chaque chômeur;

- le chômage de longue durée fonctionne comme un «piège» pour les demandeurs d'emploi qui en sont victimes, et les inscrit dans des trajectoires d'exclusion. Il est donc indispensable de favoriser un traitement préventif du chômage pour éviter que le piège ne se referme sur les plus vulnérables;

- l'indemnisation du chômage (traitement passif) et les mesures d'aide au réemploi (traitement actif) sont coûteuses, surtout en période de chômage de masse, et les SPE ont des ressources limitées. Cela suppose de développer une intervention précoce pour limiter le coût de chaque chômeur, en accélérant son retour à l'emploi.

L'objectif du profilage est de répondre à ces trois défis: en calculant dès leur inscription le risque de chômage de longue durée des demandeurs d'emploi, il permet de les orienter précocement vers un parcours personnalisé censé les empêcher de devenir CLD et les accompagner de manière adéquate vers l'emploi. Parallèlement à l'affectation des demandeurs d'emploi, le profilage joue également un rôle primordial d'optimisation des dépenses d'assurance-chômage, puisque d'une part son efficacité à réduire le temps passé au chômage pour les bénéficiaires permet de réduire d'autant les dépenses d'indemnisation, et d'autre part les dépenses actives sont ciblées sur ceux qui en retirent potentiellement le plus grand bénéfice. La question qui se pose alors est celle de la construction effective d'un tel outil: les expériences connues à ce jour ont-elles atteint ces objectifs? Ont-elles emprunté une voie unique pour y parvenir?

\section{Comment profiler?}

Selon Glynstrup et Rosholm (2005), toute technique de profilage doit poursuivre quatre objectifs : - donner une bonne estimation de la distance du chômeur au marché du travail en fonction de son niveau d'employabilité et de ses besoins en termes d'aide au retour à l'emploi ;

- cibler correctement l'affectation des ressources sur ceux qui en ont le plus besoin;

- assurer l'adéquation entre intensité de l'aide et forts risques de chômage de longue durée; 
Le modèle d'expertise (assessment by counsellor) repose sur le seul jugement d'un conseiller du SPE, qui reçoit en entretien le demandeur d'emploi, et tente d'évaluer par le biais d'un bilan interactif sa position sur le marché du travail et ses perspectives de retour à l'emploi. C'est au conseiller que revient la décision d'un potentiel accompagnement du chômeur, et le choix des mesures le cas échéant, mais il peut s'il le souhaite s'appuyer par exemple sur des questionnaires. L'avantage de cette première méthode est la possibilité de prise en compte de caractéristiques non modélisables du demandeur d'emploi (comportement, motivation, présentation de soi, etc. ), ainsi que son interactivité, qui permet dans certains cas au chômeur de jouer un rôle actif dans la décision de sa propre orientation. Mais cette méthode est aussi très coûteuse, puisqu'elle mobilise des moyens humains conséquents, surtout en période de chômage de masse. De plus, elle est soumise à un certain arbitraire, celui de la subjectivité du jugement du conseiller, elle n'échappe donc pas complètement au risque d'écrémage, et introduit une inégalité potentielle de traitement entre les demandeurs d'emploi.

Le modèle de sélection (group screening) permet de déterminer le risque de chômage de longue durée du demandeur d'emploi à partir de son appartenance objective à un groupe préalablement identifié comme 'à risque', et sur lequel sont ciblées les aides, sur la base de grilles de statistiques descriptives (par exemple les jeunes, les non-qualifiés, etc. ). Ne sont donc prises en compte que quelques caractéristiques clés du chômeur, considérées comme déterminantes, mais la faiblesse de ce modèle est du même coup d'établir un classement qui ne s'appuie pas sur la spécificité de la situation du demandeur d'emploi, et qui reste donc à un niveau très agrégé, avec le risque d'effectuer un tri biaisé, puisque le modèle ne cherche pas à calculer le risque individuel de chômage de longue durée. Reste son faible coût attractif pour les SPE. Ce profilage correspond au ciblage traditionnel des politiques de l'emploi.

Les modèles statistiques (econometric model) s'appuient sur des modèles mathématiques parfois très sophistiqués, modélisant à la fois la situation du demandeur d'emploi et celle du marché du travail sur lequel il s'inscrit, à partir du calibrage d'une série de variables influençant potentiellement sa probabilité de chômage de longue durée (cf. encadré 2). Ces modèles permettent de calculer pour tous les demandeurs d'emploi un risque global de chômage de longue durée (sous forme d'un score individuel), et donc de déterminer les chômeurs à aider prioritairement. Ces modèles ont l'avantage de l'objectivité du diagnostic (et donc de limiter l'écrémage), d'un coût relativement modeste, de pouvoir être fréquemment actualisés et donc d'être itératifs, et de cibler précisément les bénéficiaires des mesures d'accompagnement. Cependant ils dépendent fortement de la qualité des données et de leur modélisation.

( ${ }^{*}$ ) Cette typologie est celle proposée par le rapport de PLS RAMBoll MANAGEMENT (2001). Elle permet de recenser toutes les méthodes de segmentation précoce des demandeurs d'emploi (définition large du profilage). Ces catégories font aujourd'hui figure de référence, c'est pourquoi nous les présentons, mais seuls les modèles statistiques entrent pleinement dans la définition que nous retenons du profilage dans cet article.

- et utiliser une méthode suffisamment heuristique pour qu'elle permette un réajustement du diagnostic en fonction des évolutions de la situation personnelle du demandeur d'emploi et de la conjoncture du marché du travail.

Ces conditions leur font conclure qu'un outil de profilage peut jouer trois rôles différents :

- un simple profilage initial, permettant de mesurer la capacité du chômeur à retrouver ou non seul un emploi;

- un profilage itératif, consistant en un ajustement régulier du jugement en fonction des évolutions de la situation du demandeur d'emploi;

- un profilage de placement, incluant dans le diagnostic initial le parcours d'aide au retour à l'emploi, permettant de déterminer les mesures à prendre pour accompagner le chômeur.

Chaque outil de profilage peut ne consister qu'en la sélection d'une de ces trois caractéristiques, ou en une combinaison de plusieurs éléments, l'idéal étant bien sûr que la technique mise en œuvre inclue les trois dimensions: une collecte pertinente d'informations sur le chômeur et sur ses perspectives de réemploi, un outil de classement des demandeurs d'emploi en fonction de ces données régulièrement mis à jour, et une offre de services personnalisée en fonction des résultats obtenus à l'aide de l'outil de classement. En se fondant sur ces trois axes, on définit le profilage comme une procédure appliquée à une partie ou à l'ensemble des demandeurs d'emploi à un moment précoce de leur inscription au chômage, afin de déterminer leur risque individuel de chômage de longue durée et de proposer des mesures de placement adaptées à leur situation particulière, en fonction du résultat obtenu.

Or seul le profilage statistique permet a priori de répondre à l'ensemble de ces défis, car il est le seul à permettre un calcul précis du risque individuel de chômage de longue durée. Les modèles d'expertise se contentent en effet d'estimer la distance au marché du travail sans pouvoir en donner une mesure quantifiée, permettant d'évaluer l'ensemble des bénéficiaires potentiels sur une échelle unique; et les modèles de sélection ne proposent qu'un classement agrégé, en distinguant des groupes cibles éligibles aux dispositifs de politique de l'emploi, sans tenir compte de 
variables personnelles qui peuvent parfois être bien plus pertinentes pour le parcours individuel que l'appartenance à un groupe donné.

Cependant, les modèles statistiques présentent d'emblée la faiblesse de ne pouvoir intégrer que les caractéristiques modélisables des demandeurs d'emploi, et donc difficilement prendre en compte des facteurs qualitatifs ou psychologiques qui jouent un rôle pourtant reconnu dans la réussite d'une réinsertion professionnelle (SEN, 1997). C'est pourquoi il est intéressant de garder à l'esprit l'apport des modèles d'expertise, car le modèle de profilage potentiellement le plus efficace est sans doute celui qui s'appuie sur l'alliance du modèle statistique et de l'expertise du conseiller, qui permet de prendre en compte le maximum de variables pertinentes influençant la probabilité de retour à l'emploi, et de

\section{Encadré 2}

\section{Les techniques de profilage statistique}

Les régressions logistiques: la variable dépendante du modèle est le fait de devenir CLD (ou d'épuiser ses droits à l'assurance chômage). Elle est donc dichotomique (prend la valeur 1 si oui, 0 sinon). À l'aide de modèles de régression logit ou probit, on calcule l'impact statistique d'un ensemble de variables potentiellement explicatives (caractéristiques individuelles et état du marché du travail) $\left(^{*}\right)$ sur cette variable dépendante. Ceci permet d'estimer le poids de chacune de ces variables explicatives dans la probabilité finale de devenir CLD. On peut ensuite normaliser ces résultats de manière à ce que, pour chaque caractéristique, on ait un indicateur variant entre 0 et une valeur déterminée (par exemple 1000) (méthode dite de scoring), puis tracer des fonctions de répartition des risques pour certains profils types et ainsi déterminer des seuils pertinents de risque. On utilise ce calibrage pour calculer la probabilité pour chaque demandeur d'emploi de devenir CLD en entrant dans l'ordinateur ses propres caractéristiques individuelles et en faisant tourner le modèle ainsi défini. C'est ce type de modèle qui a majoritairement été retenu aux États-Unis.

Les modèles de durée: l'idée fondatrice est que la durée du chômage est une variable aléatoire qui ne peut entièrement s'expliquer par des variables identifiées (il reste une composante aléatoire qu'on ne peut pas contrôler mais qu'il faut prendre en compte dans le modèle). Pour modéliser la durée du chômage, on utilise une distribution de probabilité du type Weibull (mais d'autres, plus souples sont aujourd'hui préférées), qui permet de prendre en compte les phénomènes de dépendance d'état en modélisant les trajectoires probables de sortie du chômage. Une fois la distribution de probabilité construite, on utilise les caractéristiques individuelles du demandeur d'emploi pour la paramétrer, et ainsi estimer son risque individuel de chômage de longue durée. Le Job Barometer danois est construit sur un modèle de durée.

Les arbres de décision: le but est de segmenter la population des demandeurs d'emploi en groupes homogènes par rapport à leur risque de devenir CLD, grâce à un ensemble de variables explicatives. Pour constituer ces groupes à l'intérieur desquels les demandeurs d'emploi se ressemblent en moyenne, on construit un arbre de décision, en subdivisant les chômeurs en sous-groupes successifs en fonction de l'impact des différentes variables sur le risque de chômage de longue durée. Pour constituer un sous-groupe, on segmente les demandeurs d'emploi en fonction de la variable qui permet le mieux de construire deux sous-groupes homogènes. On détermine cette variable grâce à un algorithme construit à partir des données de cohortes passées. On réitère ce processus jusqu'à avoir des sous-groupes suffisamment homogènes (degré de ressemblance statistique au-dessus d'un seuil prédéfini) pour lesquels on connaît in fine le risque de chômage de longue durée. Pour chaque chômeur, on calcule ensuite, à partir de ses caractéristiques personnelles et du bassin d'emploi où il s'inscrit, à quel sous-groupe il appartient, et donc sa probabilité de chômage de longue durée. C'est ce type de modèle qui est actuellement expérimenté en France.

- Le choix du modèle pertinent dépend de la(les) variable(s) qui est/sont définie(s) comme variable(s) dépendante(s) (target variable) (HASLUCK, 2004). Si l'on parvient à isoler un objectif central du profilage (lutter contre le chômage de longue durée, réduire les dépenses du SPE, etc. ), on choisit cette variable et ses deux modalités possibles (oui/non) et on utilise l'un des modèles de régression logistique, qui sont les plus adaptés pour les variables binaires et discrètes (BERGER, BLACK, SMITH, 2001). Si, en revanche, on refuse cette trop grande simplification, en préférant conserver plusieurs variables dépendantes, et/ou plusieurs modalités pour chacune afin de synthétiser une information pertinente (par exemple la durée précise du chômage pour chaque demandeur d'emploi, qu'on refuse de réduire à supérieure à douze mois ou non), on construit des variables continues, qui permettent une étude plus fine. Celles-ci peuvent être analysées à l'aide de modèles de durée, qui permettent la prise en compte simultanée de toutes les variables retenues, ou d'arbres de décision, qui hiérarchisent les variables selon l'importance relative de chacune (BLACK, PLESCA, SHANNON, SMITH, 2003).

$\left(^{*}\right)$ Par exemple: caractéristiques individuelles: âge, sexe, situation familiale, lieu de résidence, origine ethnique, métier, diplômes, expérience professionnelle, projet, mobilité, etc.; état du marché du travail: bassin d'emploi, secteur professionnel, métier en tension, taux de chômage local, nombre d'entreprises du secteur, taux de recrutement, etc. Le choix de ces variables ainsi que leur pondération sont stratégiques pour construire un modèle offrant une bonne prédiction du risque individuel de chômage de longue durée, et il est intéressant de constater les différences qui existent d'un modèle à l'autre, et qui traduisent des préoccupations et priorités propres aux différents pays (DEBAUCHE, GEORGES, 2007). 
négocier le résultat du profilage entre le conseiller et le chômeur. Notre champ d'analyse se restreint donc désormais aux modèles de profilage statistique, mais ne se ferme pas complètement aux autres politiques de ciblage permettant de lutter conjointement contre le chômage de longue durée (surtout pour le cas des pays qui, à l'image des Pays-Bas, se caractérisent justement par la complémentarité du profilage statistique et de l'expertise des conseillers).

\section{Le modèle américain : le profilage statistique, ressort de la décision d'orientation}

Les États-Unis sont les premiers à avoir mis en place un modèle statistique de profilage, qui constitue aujourd'hui, après plus de dix ans d'utilisation, la politique de l'emploi la plus importante du dispositif américain. Le profilage y est considéré comme efficace du point de vue de son objectif principal: réduire les dépenses consenties au titre de l'indemnisation, en incitant les bénéficiaires à retrouver un emploi avant la fin des six premiers mois de chômage.

\section{L'idéal-type \\ du «profilage statistique pur»}

La caractéristique principale du modèle américain est la confiance qu'il accorde à l'outil technique de profilage pour optimiser l'orientation des demandeurs d'emploi ainsi que le ciblage des dépenses du SPE. Il postule ainsi qu'il est possible de résumer de manière pertinente l'information qui influence la probabilité de retour à l'emploi d'un individu à l'aide un groupe préalablement défini de variables. Ce principe traduit la croyance en une double causalité : d'abord que la durée du chômage est liée à des caractéristiques identifiables, observables et quantifiables du demandeur d'emploi, et ensuite que les relations valables pour un individu le sont également pour un autre qui présente des caractéristiques similaires, si bien qu'il est légitime d'utiliser l'observation passée pour prédire le risque futur et la position théorique d'un nouvel individu sur le marché du travail.

Ces hypothèses sont celles du profilage statistique en général, mais elles sont particulièrement importantes dans le cas du modèle américain, puisque la décision repose principalement sur elles. Dans ce modèle, il n'y a en effet que peu de place laissée à la subjectivité et à l'interprétation des conseillers du SPE, ce qui permet certes de réduire l'écrémage et les potentielles inégalités entre les demandeurs d'emploi, mais induit le risque d'une évaluation «couperet» erronée qui classe définitivement le chômeur dans une catégorie qui ne devrait pas être la sienne et donc sur un parcours inadapté à sa situation réelle.
Parmi les expérimentations connues de profilage, le modèle des États-Unis est sans conteste celui qui se rapproche le plus de cet idéal-type, par la confiance qu'il accorde aux méthodes statistiques et par la logique de coût qu'il privilégie, c'est pourquoi nous le présentons en détail ci-après. Les autres pays qui se sont directement inspirés de l'expérience américaine nuanceraient cependant ce diagnostic favorable en faveur de l'efficacité du profilage: le Royaume-Uni (1997) et le Canada (1999), ont en effet abandonné le profilage, respectivement pour cause d'inadéquation avec les caractéristiques du marché du travail(4), et car il n'était pas apprécié des conseillers; et l'Australie conserve à ses conseillers la possibilité de rectifier les résultats du profilage statistique(5).

\section{L'exemple américain: une logique instrumentale au service d'un ciblage restreint}

Les États-Unis font figure de pionniers en matière de profilage. Ils sont en effet les premiers à l'expérimenter dès les années 1980, et à en faire une obligation fédérale en 1993, avec la mise en place des Worker Profiling and Reemployment Services (WPRS), incitant tous les États à fonder leurs services de réemploi sur l'établissement de profils, dans le but affiché de réduire le coût budgétaire du SPE. Si le recours à des méthodes statistiques n'est pas obligatoire, celles-ci deviennent de fait rapidement les plus utilisées pour orienter les demandeurs d'emploi, car elles permettent un calcul précis des risques potentiels pour les chômeurs indemnisés d'épuiser leurs droits à l'assurance-chômage (vingtsix semaines) et de devenir CLD (plus de six mois de chômage).

L'expérience américaine est particulièrement intéressante, car elle valide, par le biais des WPRS, trois étapes qui permettent d'en juger comme d'un ensemble cohérent et rationnel de prestations actives de l'emploi (FARMER, 1993):

-l'utilisation d'un modèle de profilage utilisant des données quantitatives pour identifier les forts risques de chômage de longue durée;

- une procédure systématique qui définit les modalités de l'octroi de services aux demandeurs d'emploi ciblés ;

- et un mécanisme de feedback qui permet l'évaluation de l'efficacité du ciblage et des services fournis.

Par leur ampleur, les WPRS constituent les mesuresphares du SPE américain. Ils valident un processus en deux étapes, lorsqu'un demandeur d'emploi vient s'inscrire au chômage ( $c f$. schéma 1$)$ :

(4) Pour plus de d'informations concernant le Royaume-Uni, $c f$. GibBins (1997) et Wells (1998).

(5) $C f$. Lipp, 2005. 


\section{Le système américain de profilage}

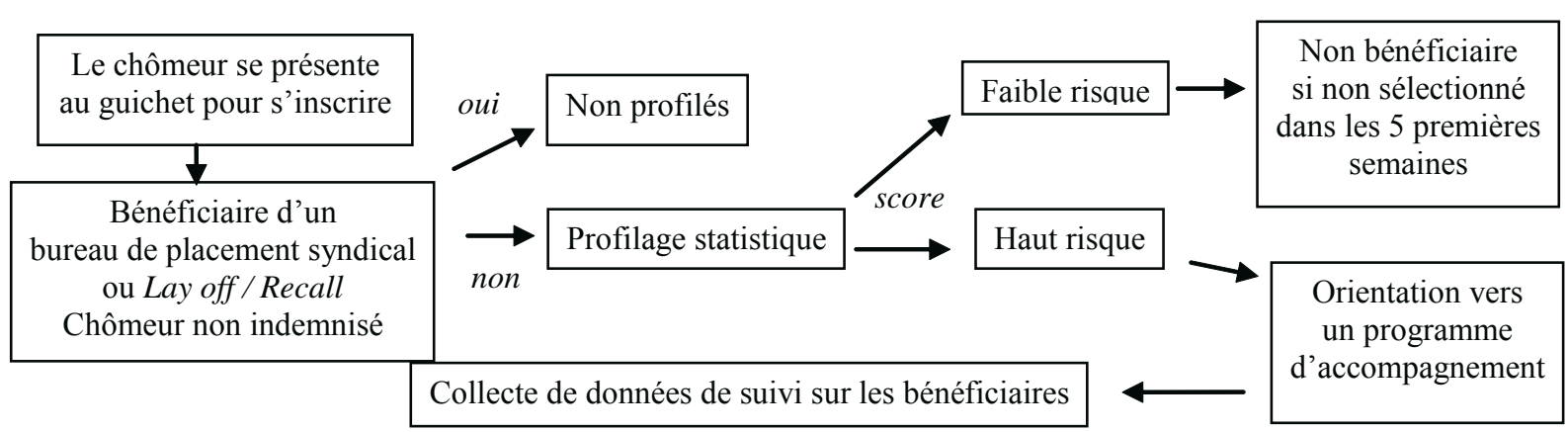

- sont d'abord exclus des aides potentielles les bénéficiaires des bureaux de placement syndicaux et du système de lay off/recall, ainsi que les demandeurs d'emploi non indemnisés. En effet, l'objectif principal du profilage est de limiter les dépenses du SPE, or seuls les demandeurs d'emploi indemnisés présentent une source potentielle d'économie par le biais d'une réduction de la durée de chômage;

- sont ensuite repérés, parmi les bénéficiaires de l'assurance-chômage, les potentiels CLD à l'aide du modèle statistique. Pour ce faire, chaque agence de l'emploi construit son propre modèle de profilage avec des données issues du marché du travail local, l'applique aux caractéristiques individuelles de chaque chômeur indemnisé, ce qui leur attribue un score (en général entre 1 et 20), puis classe l'ensemble des demandeurs d'emploi du plus fort au plus faible score. Le SPE local détermine le nombre de chômeurs qu'il peut prendre en charge en fonction de ses dotations budgétaires, assurées par le gouvernement fédéral (6), et choisit les demandeurs d'emploi à placer dans l'ordre décroissant des scores, qui sont donc les seuls déterminants de l'aide à laquelle ils ont ou non droit (EBERTS, O'LEARY, 2003).

La présélection permise par le profilage exclut la majeure partie des demandeurs d'emploi, qui, s'ils ne sont pas retenus par le modèle de profilage dans les cinq premières semaines d'indemnisation, ne pourront plus bénéficier de mesures d'aide. De plus, les faibles dotations budgétaires conduisent à ne prendre effectivement en charge que $20 \%$ des demandeurs d'emploi potentiellement éligibles aux aides (environ 20500 demandeurs d'emploi par an).

(6) Dotations budgétaires qui restent très modestes. En effet, le budget total attribué aux WPRS par le gouvernement fédéral s'est élevé à 1,64 M dollars pour l'année 2005. À titre de comparaison, le budget alloué par la France au suivi personnalisé des demandeurs d'emploi (PARE) est de 1 milliard d'euros par an (1250 $\mathrm{m}$ dollars). De manière générale, les dépenses actives aux États-Unis sont très faibles comparées à l'Union européenne $(0,13$ point de PIB en 2005 contre 0,9 pour la France, OCDE, 2007).
Ainsi, même si le profilage est appliqué à l'ensemble des chômeurs indemnisés, il ne permet le placement que d'un faible nombre d'entre eux (environ $5 \%$ de l'ensemble des demandeurs d'emploi). La conséquence principale du nombre limité de places dans les programmes est qu'il fait de la qualité de la mesure de la probabilité d'épuisement des droits le point clé du système. C'est pourquoi l'outil essentiel du profilage américain est sans conteste le modèle statistique utilisé (BERGER, Black, Noel, SMITH, 2003).

Chaque modèle s'appuie sur une architecture comparable: un volet offre, prenant en compte les caractéristiques individuelles (dans la limite de la législation sur les droits civiques qui interdit le recours aux variables personnelles que sont l'âge, le genre et l'origine ethnique (7)); et un volet demande, qui correspond aux conditions économiques du marché du travail local. La probabilité assignée à chaque chômeur est une moyenne pondérée de l'effet de chacune de ces variables sur les chances qu'il épuise ses droits. Initialement, l'USDOL(8) avait recommandé aux États de recourir à sept variables clés(9); mais aujourd'hui, suite à leur évaluation, les modèles statistiques se sont grandement complexifiés. À titre d'exemple, celui du Texas utilise aujourd'hui quarante-quatre variables différentes dans son modèle de régression logistique (EBERTS, O'LeARY, WANDNER, 2002).

Ceci justifie qu'une large majorité des évaluations se centre quasi exclusivement sur l'efficacité mathématique des modèles (c'est-à-dire sur la justesse de la prévision statistique), plus que sur les

(7) Mais une analyse comparative des modèles lorsqu'on inclut ces variables personnelles montre que leur effet sur le pouvoir prédictif du modèle de profilage est en général très faible (US DEPARTEMENT OF LABOR, 1994).

(8) US Departement of Labor.

(9) Le bénéfice du lay off/recall, l'appartenance à un bureau syndical d'embauche, l'éducation, la durée d'emploi, le changement d'emploi dans l'ancien secteur, le changement d'emploi dans l'ancienne profession, et le taux de chômage local. 
effets concrets du profilage sur le marché du travail américain. Les deux points essentiels sur lesquels se focalisent ces évaluations sont l'efficacité du ciblage permis par les modèles, et la rentabilité du système en termes de coût pour l'assurance-chômage. Très peu d'études adoptent comme point central les effets du profilage sur l'emploi des anciens bénéficiaires, et celles qui sont réalisées ne peuvent s'appuyer que sur trois variables (qui sont celles collectées par le SPE au cours du parcours individualisé de retour à l'emploi): le nombre de bénéficiaires pourvus d'un emploi au cours des quatre trimestres suivants, leur salaire par rapport au salaire antérieur à la demande de prestations et si le nouvel emploi représente un changement de branche d'activité (OCDE, 2000). Et comme on ne collecte pas de données de suivi sur les chômeurs qui n'ont pas trouvé d'emploi, il est difficile d'obtenir des résultats pertinents.

Malgré cela, Berger et al. (2003) montrent que les bénéficiaires profitent d'une baisse de la durée moyenne de chômage indemnisé(10), du montant des indemnités versées et d'une hausse des salaires moyens au cours de l'année suivante. Ces effets sont nuancés par le fait qu'il n'existe pas de relation systématique entre impact estimé du programme et probabilité estimée de chômage de longue durée, et que ces bons résultats s'estompent à long terme. Une étude plus ancienne (HIPPLE, 1997) concluait que 70 à $80 \%$ des bénéficiaires étaient occupés au bout de deux ans et touchaient $80 \%$ de leur rémunération antérieure. Mais il manque à ces évaluations un moyen de calculer rigoureusement ce qui serait arrivé aux demandeurs d'emploi ciblés s'ils n'avaient pas bénéficié des services, ce qui rend ces travaux relativement incomplets.

Il en va de même pour les études qui tentent d'analyser le type de service le plus efficace proposé par le SPE aux bénéficiaires: si toutes concluent que les profilés ont bénéficié de beaucoup plus de services que ceux qui n'ont pas été orientés vers les WPRS, elles soulignent aussi l'absence de véritable cohérence du programme puisqu'aucune nouvelle mesure d'aide n'a été mise en œuvre en même temps que les WPRS. La faiblesse majeure est l'absence de lien clairement établi entre le profilage initial et l'offre de services. Les WPRS ne sont donc pas un profilage de placement. Une tentative avait vu le jour pour pallier ce manque(11), mais elle a rapidement avorté.

(10) En 2005, l'US Departement of Labor estimait que 51\% des bénéficiaires des WPRS épuisaient effectivement leurs allocations, contre $54 \%$ des non-bénéficiaires.

(11) Le Frontline Decision Support System (FDSS), initié en 2000 en Géorgie (mais abandonné en 2003), qui tentait de lier explicitement le profilage à l'offre de services, c'est-à-dire d'associer automatiquement au score obtenu les mesures à prendre pour aider les demandeurs d'emploi (O'LEARY, 2006).
Reste que pour les deux objectifs mis en avant, le profilage statistique semble être un succès: en termes de ciblage, l'accélération du retour à l'emploi est significative (en moyenne les WPRS réduisent la durée de chômage de 2,2 semaines) tout comme en termes de coût (le SPE économise 143 dollars par chômeur profilé, et ceux-ci touchent un supplément de salaire de 1050 dollars la première année, cf. Berger et al., 2003). Bien que ces évaluations ne permettent en rien de distinguer l'effet propre du profilage de celui des services reçus en aval, on peut retenir deux atouts du système américain: il est économique, et les modèles statistiques sont efficaces. Mais la rançon de ces succès est une trop forte focalisation sur ces deux points, c'est-à-dire une seule critique technique du profilage [l'étude de BlACK et al., (2003) par exemple, ne fait que des recommandations économétriques pour parfaire les modèles mathématiques, eux-mêmes pourtant critiqués ( $c f$. Olsen, DeCKer, 2001)], et un intérêt trop soutenu pour le problème du coût, au détriment de la qualité de l'accompagnement.

Le modèle américain repose sur un outil statistique relativement perfectionné, ce qui fait à la fois sa force (un ciblage efficace et de substantielles économies pour le SPE) et sa faiblesse, puisque la qualité des services proposés aux demandeurs d'emploi s'en ressent et que les agents des services locaux de l'emploi sont en partie dépossédés de leur expertise (ce sont les résultats du modèle statistique qui décident automatiquement de faire bénéficier ou non un individu de mesures d'accompagnement, de surcroît sans préciser lesquelles). Derrière le profilage statistique pur à l'américaine se cache donc une tentation «instrumentaliste», qui laisse à l'outil profilage la possibilité de coordonner seul les actions des agents, sans qu'aucune marge de négociation ne demeure. Le risque inhérent à ce choix est d'une part que les modèles de profilage ne soient pas à la hauteur de leur mission, et de l'autre que le SPE se voie relégué à un rôle subalterne dans la mise en œuvre de la politique de l'emploi.

\section{Le modèle néerlandais: le profilage, outil d'aide à la segmentation des demandeurs d'emploi}

Face au modèle fondateur américain et à son apparente efficacité, plusieurs pays européens se sont saisis du profilage statistique, et ont tenté de l'adapter à leurs besoins et aux contextes institutionnels particuliers qui sont les leurs. Parmi eux, les Pays-Bas présentent la palette complète de l'expérimentation du profilage comme politique de l'emploi: mise en place précoce, utilisation à grande échelle, évaluation, et abandon au profit d'une segmentation «manuelle» faute d'efficacité. 


\section{L’idéal-type du «profilage négocié »}

La plupartdes pays qui ont expérimentéle profilage ont tous choisi de ne le considérer que comme un outil d'aide à la décision pour les conseillers. C'est l'entretien direct avec le demandeur d'emploi qui détermine son orientation, le modèle de profilage offrant la possibilité d'une information supplémentaire sur le parcours optimal que le conseiller peut proposer au chômeur.

Ceci peut s'expliquer par l'objectif assigné au profilage: contrairement aux États-Unis, dans de nombreux cas, ce n'est pas tant le risque de chômage de longue durée lui-même qui intéresse le SPE, que la possibilité de segmenter les demandeurs d'emploi dans différents parcours d'aide à la réinsertion. En ce sens, le risque statistique est une information sur la catégorie pertinente dans laquelle classer l'individu, et non la variable déterminante de son orientation. Le principe technique sur lequel reposent la segmentation et l'individualisation des parcours est le ciblage (targeting), qui vise l'allocation efficace de l'offre de services, et non le profilage, dont le rôle est d'identifier les besoins des demandeurs d'emploi en fonction de leur risque individuel (Frölish, Lechner, Steiger, 2004; Hasluck, 2006)(12). Ainsi, dans le premier cas on se place du point de vue de l'intérêt collectif, du SPE, (et donc le rôle du conseiller est mis en avant); tandis que dans le second c'est la responsabilité individuelle qui prime.

Deux systèmes pourraient parfaitement illustrer cet idéal-type: les Pays-Bas et le Danemark. Nous choisissons le premier en raison de son antériorité, qui permet d'avoir des évaluations plus approfondies(13).

\section{L'exemple néerlandais: un modèle de concertation où le rôle du profilage est en recul}

Le premier atout du modèle néerlandais de profilage est, tout comme pour les États-Unis, sa relative ancienneté (1999). Premier pays européen à avoir mis en place un système rationalisé et uniformisé de profilage, il sert souvent d'exemple phare aux partisans d'une généralisation de la méthode à tous les pays soumis à la SEE. Cependant, les récentes critiques à l'encontre de l'efficacité du modèle statistique utilisé en font un sujet de débat qui déborde les frontières des Pays-Bas.

(12) Notons que le profilage de placement se rapproche en ce sens du ciblage, puisqu'il cherche à combiner le calcul du risque individuel avec l'affectation optimale des demandeurs d'emploi dans les différents programmes d'aide. Les deux objectifs ne sont donc pas irréconciliables.

(13) Pour une présentation du modèle danois, $c f$. Hammer, Rosholm, Svarer, 2004; Delautre, Georges, 2007.
L'originalité des Pays-Bas est d'avoir d'emblée compris les risques inhérents à une pratique purement formelle du profilage, et de l'avoir complétée - aujourd'hui remplacée(14) - avec l'expertise des agents du SPE, dont le rôle, loin d'avoir été minimisé par l'introduction d'un modèle statistique de profilage, en est sorti renforcé. L'objectif de base du profilage aux Pays-Bas est ainsi «d'accompagner le conseiller dans son travail d'expertise avec un nouvel outil à la décision et non de se substituer à son appréciation » (Delarue, 2005). Les objectifs du Kansmeter sont ainsi d'emblée institutionnels puisqu'il peut améliorer les appariements entre les services disponibles d'aide au retour à l'emploi et le chômeur en position difficile sur le marché du travail, ainsi que la transparence de l'action du SPE chargé de rationaliser son action (HERBILLON, 2004). La mise en place du profilage poursuit donc une efficacité tant statistique qu'organisationnelle.

Le Kansmeter s'appuie sur des variables synthétisant les caractéristiques personnelles et l'employabilité du chômeur, ainsi que l'état du marché du travail. Le calcul final est relativement basique, sous la forme d'une moyenne pondérée des variables, issue de l'observation empirique et non d'un étalonnage sophistiqué, à partir de l'attribution de points à chaque critère évalué, selon qu'il est jugé «bon», «satisfaisant», «moyen» ou «mauvais ». Le modèle statistique néerlandais est donc loin des raffinements théoriques américains, d'autant plus que la complexité statistique du modèle tend à décroître au fur et à mesure des réformes issues de l'évaluation, suivant ici un chemin inverse aux perfectionnements mathématiques des modèles américains, - et aux trajectoires habituelles des dispositifs de politique de l'emploi qui tendent plutôt généralement à se complexifier.

Ce profilage statistique a lieu lors de l'entretien d'inscription du chômeur, qui permet de faire une évaluation globale de sa situation (le Kwint) ( $c f$. schéma 2). Ici, le jugement du conseiller reste relativement en retrait par rapport au modèle, puisque les questions sont majoritairement fermées. À l'issue de ce premier contact, les chômeurs sont classés en trois catégories: $\mathrm{C} 1$ : distance faible au marché du travail (recherche autonome), $\mathrm{C} 4$ : distance très importante (programmes d'aide sociale), et une catégorie pour tous les demandeurs d'emploi qui ne rentrent ni dans l'une ni dans l'autre, et qui sont renvoyés à un entretien de diagnostic approfondi. Si le résultat statistique ne correspond pas à ce qu'il observe, le conseiller a la possibilité de le «rectifier», en corrigeant la note donnée par l'ordinateur s'il la juge erronée. Cette option de correction est présente tout au long du processus d'accompagnement vers l'emploi, puisque le conseiller est libre de

(14) L'utilisation du Kansmeter a été suspendue à l'automne 2006. 
Schéma 2

Le système néerlandais initial de profilage

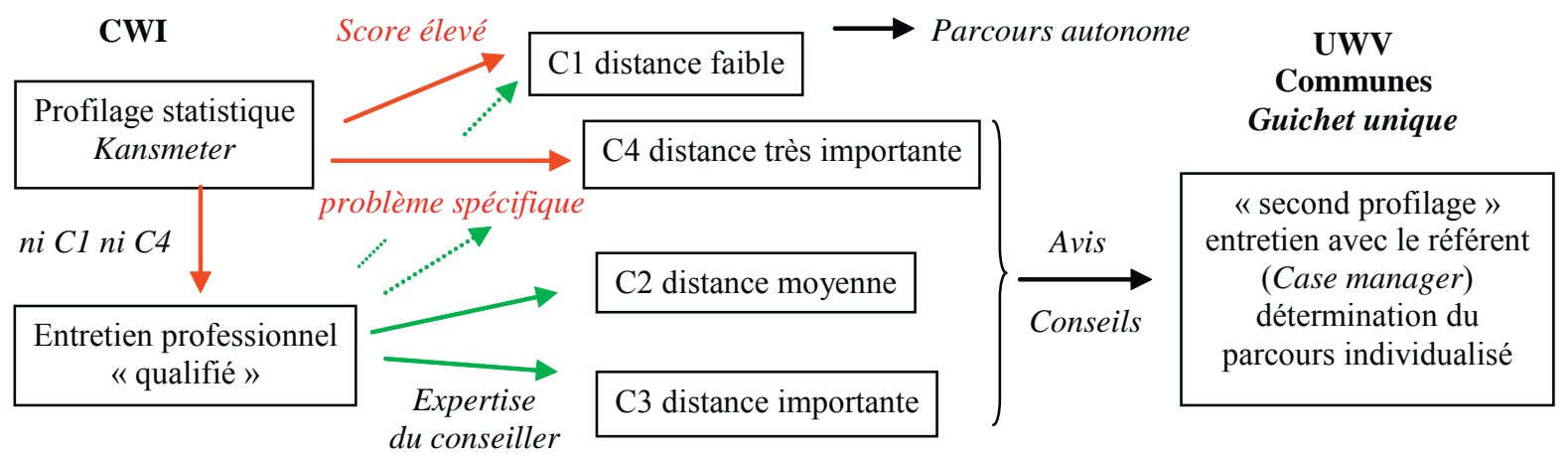

D'après HerBiLlon, 2004

procéder à un réexamen de la note obtenue à n'importe quel moment. Il y a donc dès le premier entretien une marge de «négociation» entre le chômeur et le conseiller concernant le résultat du profilage, et donc le chemin emprunté par le chômeur pour se rapprocher de l'emploi.

Cette souplesse dans l'utilisation de l'outil est renforcée pour ceux qui n'ont pas été classés lors du premier entretien. En effet, leur score «moyen» est jugé non significatif pour pouvoir directement conclure à un choix de parcours. Les demandeurs d'emploi concernés sont donc convoqués à un entretien approfondi, où le conseiller ne recourt plus au Kansmeter. Le résultat statistique obtenu grâce au profilage n'est donc qu'une première étape censée aider le conseiller à décider de la marche ultérieure à suivre. Le profilage statistique permet surtout un «dégrossissement» rapide en début de parcours, pour favoriser l'orientation rapide des deux types « extrêmes » de demandeurs d'emploi (les autonomes $\mathrm{C} 1$ et les pris en charge spécifiques C4). Il apparaît ainsi comme «le fruit d'une démarche raisonnée et équilibrée visant à structurer un processus sans pour autant le figer dans un cadre statistique rigide. [...] En ce sens, il est davantage question d'expertise outillée que de modèle statistique en tant que tel» (Herbillon, 2004).

À l'issue du second entretien dit «qualifié», qui a lieu si possible dans les huit jours, les demandeurs d'emploi rejoignent l'une des quatre catégories (C2 : distance moyenne [ $<1$ an], C3 : distance importante [entre 1 et 2 ans]), ce qui détermine leur «parcours d'intégration» vers l'emploi, c'est-à-dire à la fois la nature et l'intensité des services d'accompagnement qu'ils vont se voir proposer, mais aussi l'insti- tution qui va les prendre en charge (15) (au final, la répartition entre les différentes catégories est assez équilibrée; par exemple en mai 2005, sur 695000 profilés, $17,4 \%$ étaient classés en $\mathrm{C} 1,20,1 \%$ en $\mathrm{C} 2$, $28,8 \%$ en $\mathrm{C} 3$ et $27,2 \%$ en C4, OcDE, 2006).

Cependant, l'efficacité de ce système a récemment été remise en cause, en raison notamment d'un faible rendement prédictif du modèle statistique (TNo-ArbeID, 2003). En effet, bien que, comme le notent les premières évaluations (BUNT, VAN Pijkeren, Bouwmeester, 2000), la plupart des objectifs organisationnels aient été $a$ priori atteints (transparence, rationalisation et uniformisation de l'action préventive et de l'offre de services), le profilage néerlandais semble pécher par sa faible fiabilité en termes de prévision du devenir des demandeurs d'emploi, puisqu'il ne permet d'estimer correctement la sortie du chômage que dans $61 \%$ des cas, $11 \%$ des chômeurs quittant le chômage plus tôt que prévu par le modèle, et $28 \%$ plus tard.

Cette limite technique induit l'une des limites théoriques du profilage: "This policy carries with it an important risk: the unemployed that are initially classified as having good job prospects may also become long-term unemployed. Therefore, labour market policies should not rely exclusively on profiling at the start of an unemployment spell, but should also adopt supplemental policies - those that, for instance, encourage search activities of all workers that have spent a certain length of time in unemployment" (Koning, VAN LeUvensteiJn,

(15) Le SPE néerlandais (le CWI, Centre pour l'emploi et les revenus) ne se charge plus que des $\mathrm{C} 1$ (moins de $20 \%$ des demandeurs d'emploi), et adresse les autres catégories de chômeurs aux «instances d'allocation» qui sont l'assurancechômage (UWV, Institution d'assurance du personnel salarié) et les communes, selon le principe de «qui paie décide». 
Schéma 3

Le nouveau système néerlandais de profilage ( $A B$ Routering)

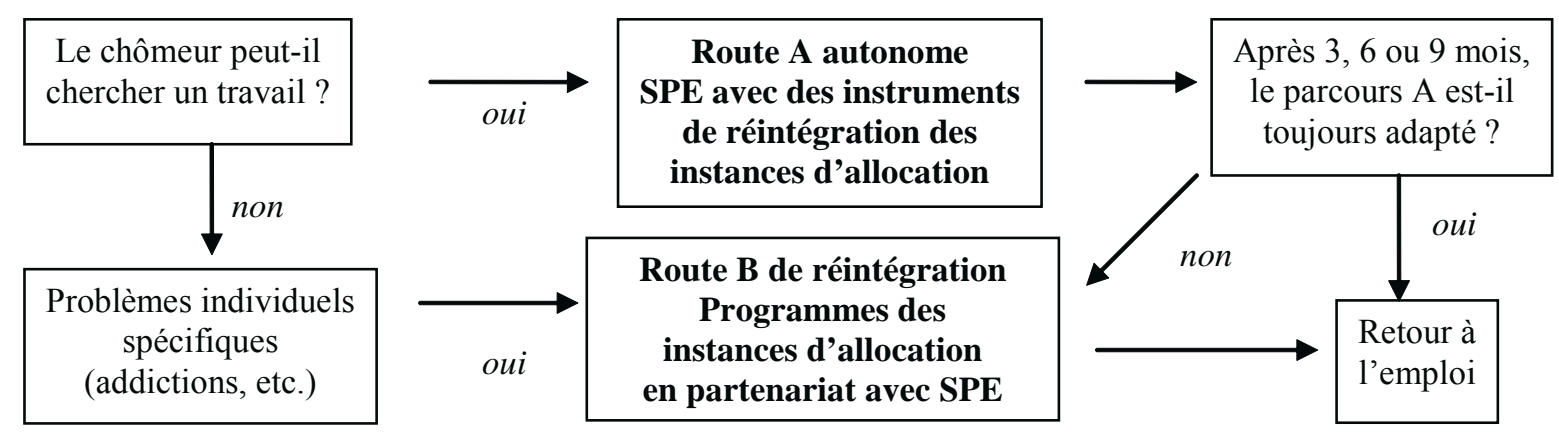

D'après FreTZ, 2005

2000)(16). Une mauvaise orientation précoce des demandeurs d'emploi risque ainsi de les enfermer dans une catégorie à laquelle ils ne devraient pas appartenir, et donc de les contraindre à un parcours d'accompagnement inadapté, refermant sur eux le piège de la dépendance d'état.

C'est pourquoi le SPE néerlandais a récemment réformé le dispositif en retardant le moment du profilage, afin de vérifier au préalable s'il n'est pas possible de placer directement les chômeurs sur le marché du travail, en attendant au moins trois mois avant de mobiliser les instruments de la politique de l'emploi (De Koning, 2004). Lors de l'inscription, un simple diagnostic de situation professionnelle est effectué, et le profilage est affiné si le chômeur ne parvient pas à se réinsérer seul sur le marché du travail, afin de répondre aux exigences d'un profilage itératif.

En outre, malgré une acceptation relativement bonne de l'outil par les conseillers, nombre de problèmes institutionnels ont été mis à jour, notamment dus à la séparation - et à la rivalité - entre CWI et instances d'allocation, ce qui conduit la plupart du temps à un «double profilage» coûteux, les seconds refusant de faire confiance au diagnostic du premier et procédant à une nouvelle évaluation (OCDE, 2006). Ce surcoût justifie l'expérimentation actuelle d'un nouveau système, l'AB Routering, qui supprime les quatre catégories précédentes pour les remplacer par deux parcours-types (A et B), l'un d'autonomie dans la recherche d'emploi

(16) «Cette politique s'accompagne d'un risque important: celui de voir certains chômeurs qui sont dans un premier temps classés comme proches de l'emploi devenir quand même chômeurs de longue durée. C'est pourquoi les politiques de l'emploi ne doivent pas s'appuyer exclusivement sur le profilage en début de période de chômage, mais également intégrer d'autres types de prestations - comme par exemple celles qui appuient la recherche d'emploi de tout chômeur qui a déjà passé un certain temps au chômage». (parrainé par le CWI), l'autre d'aide renforcée (sous la tutelle des instances d'allocation) ( $c f$. schéma 3). Le passage de l'un à l'autre peut constamment être décidé selon l'évolution de la situation du chômeur, et le CWI et les instances d'allocation peuvent désormais offrir leurs services simultanément aux demandeurs d'emploi, afin de pratiquer un profilage actif. Le recours au Kansmeter devient optionnel : il est un simple outil auquel le conseiller peut ou non recourir pour prendre sa décision d'orientation, ce qui correspond en pratique à son abandon.

Le nouveau système de profilage doit permettre de répondre aux cinq critiques majeures qui ont été adressées à l'ancien (FrETZ, 2005):

- une attention trop soutenue portée à la position théorique du demandeur d'emploi sur le marché du travail plutôt qu'à sa situation réelle;

- le risque de ne s'attacher qu'aux obstacles qui limitent le retour à l'emploi plutôt que d'exploiter les opportunités offertes et les compétences du chômeur;

- un jugement «couperet» ne reposant que sur un diagnostic initial;

-l'exclusion ex ante de nombreux demandeurs d'emploi de programmes d'aide qui pourraient pourtant leur être bénéfiques;

- et la synchronisation inadéquate entre le CWI et les instances d'allocation.

Le recul actuel du modèle de profilage statistique au bénéfice de la négociation directe entre conseiller et chômeur a tendance à renforcer l'idée d'un modèle d'expertise outillée; mais, paradoxalement, l'insistance sur la place que doit conserver l'entretien individuel tend à annihiler progressivement le rôle que sont censés jouer les modèles statistiques. Maintenant que le système $A B$ Routering est effectivement mis en place, peut-on encore parler de profilage alors qu'une large majorité des demandeurs d'emploi sont placés en parcours d'autonomie (A), quand les autres (B) se voient proposer des mesures qui relèvent davantage de l'aide sociale que des 
politiques de l'emploi? Les Pays-Bas, qui avaient jusqu'alors fait figure de modèle en Europe concernant la question du profilage remettent ici en cause l'idée même de l'utilité d'un profilage statistique. Il est donc légitime de s'interroger sur la pérennité du profilage statistique, quand ceux qui l'ont expérimenté un certain temps l'ont ensuite abandonné pour cause d'inefficacité (17).

\section{Efficacité et limites du recours au profilage}

Les deux exemples présentés ci-dessus suggèrent que non seulement les deux modèles idéal-typiques s'opposent dès l'origine quant à leur utilisation du profilage statistique, mais surtout que cette différence tend à se creuser. En effet, d'un côté, les États-Unis raffinent de plus en plus leurs modèles économétriques afin de renforcer l'efficacité de l'outil, tandis que de l'autre, les Pays-Bas abandonnent progressivement le recours systématique à un modèle statistique pour privilégier l'approche concertée entre le conseiller et le demandeur d'emploi. Deux points méritent alors un éclaircissement: pourquoi les outils statistiques mobilisés ont-ils des efficacités différentielles selon les systèmes où ils sont mis en place; et comment expliquer les choix respectifs des différents pays face au recours potentiel à des techniques de plus en plus sophistiquées, à l'image des outils de profilage.

\section{Une différence d'efficacité qui repose sur des besoins contrastés pour lutter contre le chômage}

L'observation des résultats obtenus à l'aide des outils de profilage dans les différents pays qui l'ont implémenté pose immédiatement la question de la différence d'efficacité constatée entre les modèles américain et néerlandais ou danois. En effet, les évaluations américaines mettent en avant l'amélioration des performances financières du système d'indemnisation grâce aux WPRS qui accélèrent le retour à l'emploi, quand les Pays-Bas critiquent les erreurs récurrentes d'orientation des demandeurs d'emploi imputées au profilage, et le Danemark l'absence de valeur ajoutée par rapport au travail «classique» d'orientation effectué par les conseillers. D'emblée, on comprend que les objectifs du recours au profilage ne sont pas les mêmes dans les deux modèles, ce qui justifie une utilisation différenciée de l'outil, mais n'explique cependant pas l'efficacité contrastée d'une même technique à l'œuvre dans des systèmes distincts. Pour ce faire, plusieurs hypothèses ont été avancées.

(17) Les Pays-Bas, donc, mais aussi le Danemark, le RoyaumeUni et le Canada.
D'aucuns mettent d'abord en avant la faiblesse mathématique des modèles construits dans les pays où les résultats ont été décevants. Il est clair, par exemple, qu'il existe un écart qualitatif important entre certaines méthodes statistiques retenues aux États-Unis, qui reposent sur une modélisation complexe, et le modèle néerlandais qui fonctionne comme une simple moyenne pondérée. Cependant, l'exemple danois remet en cause la pertinence de cette hypothèse pour justifier de l'efficacité différentielle de l'outil statistique, car la marge d'erreur du modèle de durée qui y a été testé reste élevée malgré sa formalisation complexe(18), ce qui explique d'ailleurs en partie pourquoi il a été abandonné. La qualité de l'appareillage statistique, si elle participe évidemment de l'efficacité de l'outil, ne peut donc à elle seule garantir la bonne orientation des demandeurs d'emploi, assurant à ces derniers un retour à l'emploi plus rapide, et du même coup pour le SPE une maîtrise des dépenses d'indemnisation.

Au-delà du design des outils proprement dit, des hypothèses qui tiennent davantage à la nature du chômage et au rôle traditionnellement dévolu aux politiques de l'emploi dans chacun des modèles peuvent également permettre de mieux comprendre pourquoi dans certaines configurations le profilage fonctionne, et pourquoi dans d'autres il ne marche pas.

En effet, les États-Unis apportent traditionnellement une aide minimale aux demandeurs d'emploi, en partie car le marché du travail y est très flexible, ce qui permet à de nombreux chômeurs de se réinsérer rapidement par leurs propres moyens, sans avoir besoin pour cela d'un accompagnement spécifique. Le nombre de places dans des programmes d'accompagnement est donc très limité, ce qui rend le tri de ceux qui ont besoin d'en bénéficier plus aisé, car les mesures ne concernent que ceux qui sont très éloignés de l'emploi, et qu'il est plus facile de repérer du fait de caractéristiques facilement identifiables.

En revanche, dans nombre de pays européens, dont les Pays-Bas et le Danemark, la plupart des chômeurs ont besoin d'une aide individualisée à la recherche d'emploi, notamment parce que le risque de devenir et de rester chômeur de longue durée y est plus élevé et plus aléatoirement réparti(19). Il est donc nécessaire d'orienter chacun vers un

(18) Le profilage danois s'appuie sur des modèles de durée qui diffèrent selon les catégories de public (huit) et les régions (quatorze), si bien qu'il en existe cent douze différents (chacun incluant entre cent dix et cent cinquante paramètres, soit plus de 14000 au total).

(19) Le chômage de longue durée est en moyenne beaucoup plus élevé dans de nombreux pays d'Europe qu'aux États-Unis: $60,9 \%$ à six mois pour l'Union européenne à quinze contre $17,6 \%$ aux États-Unis ; $44,2 \%$ à douze mois pour 1'Union européenne à quinze contre $10 \%$ aux États-Unis en 2006 (OCDE, 2007). 
programme qui lui est adapté afin de pallier ce risque, et le profilage doit non seulement sélectionner les bénéficiaires, mais encore aider à choisir pour chacun l'aide la plus efficace. Les résultats du profilage sont d'ailleurs évalués à partir de variables telles que le taux de retour à l'emploi après participation à un programme, ou la qualité de l'emploi retrouvé, variables qui dépendent sans doute davantage du programme suivi que de la qualité du profilage initial. Il est donc difficile de démêler l'effet propre du profilage de celui des politiques de l'emploi mobilisées, ce qui complique la tâche d'évaluation. Il est donc possible que, dans certains cas, on impute au profilage certains mauvais résultats qui sont en fait ceux des programmes auxquels a participé un chômeur.

D'un côté, donc, le rôle assigné au profilage n'est que de trier une petite minorité de demandeurs d'emploi en grande difficulté, alors que de l'autre, il doit orienter chaque chômeur vers le parcours qui lui convient. Il y a donc une véritable différence de nature entre le profilage statistique pur à l'américaine et le profilage négocié à la néerlandaise: le premier ne doit effectuer qu'un classement, quand le second doit garantir le bon aiguillage des demandeurs d'emploi et donc inclure en son sein l'offre de services, ce qui en termes de construction effective de l'outil est bien plus difficile à réaliser, puis à évaluer.

L'efficacité du profilage dépend donc conjointement des besoins particuliers des différents systèmes en fonction des caractéristiques de leur marché du travail, et des choix qu'ils effectuent en définissant les objectifs que le profilage doit aider à atteindre. Selon les modalités administratives de gestion et d'orientation des demandeurs d'emploi, et les ressources financières à leur disposition, les SPE ont intérêt ou non de recourir à un modèle de profilage, car selon les configurations, celui-ci sera ou non potentiellement efficace.

\section{De l'utilité contextuelle du profilage}

L'exemple du Royaume-Uni est particulièrement intéressant pour comprendre les conditions d'efficacité et les limites du recours au profilage. Dès 1994, un modèle de profilage, le Early Identification Pilot Project, a été testé dans sept Jobcentres, dans la droite ligne des expérimentations américaines. Outre les critiques d'ordre technique qui ont été adressées au modèle, les plus intéressantes ont sans doute été celles qui se sont appuyées sur les caractéristiques particulières du marché du travail britannique, jugées incompatibles avec le principe même du profilage. Le fait que le chômage y soit essentiellement transitoire, avec un taux de turnover élevé limite en effet les possibilités de détection des forts risques de chômage de longue durée (WELLS, 1998). La rapidité moyenne de sortie du chômage est élevée pour tous les demandeurs d'emploi, quelles que soient leurs caractéristiques individuelles, et l'enfoncement dans le chômage de longue durée est aléatoirement réparti, sans qu'il soit possible de détecter des régularités statistiques. Ainsi, la vitesse de sortie varie peu en fonction des caractéristiques personnelles, qu'il s'agisse de l'âge, du sexe, de la région de résidence, etc. Des régularités causales étant quasi inobservables, il n'est pas étonnant que le profilage n'ait pas fait ses preuves au RoyaumeUni, où on lui a préféré une activation intensive en début de parcours pour tous, afin que les chômeurs restent proches de l'emploi sur un marché du travail où la variété des offres multiplie les possibilités de débouchés, et donc de sortie rapide.

Le cas britannique souligne que le profilage n'est pas forcément la panacée. Son efficacité est située, c'est-à-dire qu'elle dépend avant tout des conditions institutionnelles et conjoncturelles dans lesquelles il est mis en place. D'une part, d'un point de vue conjoncturel, le profilage présente surtout un intérêt pour des pays qui ont un fort taux de chômage de longue durée, assorti d'un phénomène d'hystérèse, et où les chômeurs de longue durée ont des points communs qui pourraient être à l'origine de leur discrimination sur le marché du travail. D'autre part, le contexte institutionnel dans lequel on implante ce type d'outil est essentiel. En effet, on constate que partout l'introduction du profilage s'est accompagnée de réformes profondes des systèmes d'indemnisation et d'accompagnement des demandeurs d'emploi (guichet unique, externalisation, décentralisation, etc. ), réformes qui ont suivi des orientations relativement différentes. À titre d'exemple, on observe que le profilage a renforcé le pouvoir d'orientation du SPE au Danemark, alors qu'il a légitimé l'externalisation massive du placement aux Pays-Bas (Georges, 2006). On peut ainsi formuler l'hypothèse que les réformes entourant la mise en place du profilage auront également un impact important sur son efficacité.

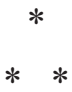

Finalement, le profilage n'est-il pas l'arbre qui cache la forêt? Actuellement au cœur des débats européens sur les nouvelles politiques de l'emploi à mettre en œuvre, il n'est en fait que le premier pas vers une réforme plus globale destinée à fonder l'aide au retour à l'emploi sur la nouvelle logique de parcours, celle de l'accompagnement individualisé et du suivi personnalisé des demandeurs d'emploi. Pour cela, le profilage peut constituer une étape préliminaire, permettant de segmenter les chômeurs sur les différents parcours. Mais le modèle «combiné» (d'expertise outillée) développé en Europe par de plus en plus de pays (Allemagne, France, Finlande, etc. ) semble ne fonctionner que dans une version très allégée, à 
l'image de celles du Danemark et des Pays-Bas, qui ont rejeté le profilage tout en conservant l'essentiel: les réformes institutionnelles de fond.

Àce titre, seuls les États-Unis ont en fait réellement développé et conservé un vrai modèle statistique de profilage au cœur de l'orientation des demandeurs d'emploi, soulignant ses qualités d'objectivité, et d'outil efficient pour l'allocation des ressources du SPE. Les caractéristiques particulières du marché du travail américain expliquent en grande partie cette efficacité comme technique de détection et d'orientation des forts risques de chômage de longue durée, qui est pour l'instant restée une relative utopie dans les autres pays qui ont choisi de l'implanter. Cette réussite du profilage Outre-Atlantique s'inscrit dans le cadre plus large du développement d'outils de plus en plus sophistiqués dans le champ des politiques de l'emploi, notamment sur le plan de la modélisation statistique, dans l'optique de fournir des évaluations raffinées. Il y a sans doute un écart considérable dans la conception des politiques de l'emploi telles que les promeuvent les États-Unis et les pays d'Europe, lié notamment à l'utilisation traditionnelle de l'économétrie pour mener à bien expérimentations et évaluations. Cette confiance américaine dans la technique explique également en partie les réussites enregistrées par le profilage, qui a surtout déçu ailleurs (GeORGEs, 2007).

\section{Bibliographie}

Benarrosh Y. (2000), «Tri des chômeurs: le nécessaire consensus des acteurs de l'emploi», Travail et Emploi, $\mathrm{n}^{\circ} 81$, pp. 9-26.

Berger M., Black D., Noel B., Smith J. (2003), "Is the Threat of Reemployment Services More Effective than the Services Themselves? Evidence from Random Assignment in the UI System, Experimental Evidence from the UI System", American Economic Review 94 (4) : 1317-1327.

Berger M., Black J., Smith J. (2001), "Evaluating Profiling as a Means of Allocating Government Services", mimeo.

Black D., Plesca M., Shannon S., Smith J. (2003), Profiling UI Claimants to Allocate Reemployment Services: Evidence and Recommendations for States, Final Report.

Bunt S., Van Pijkeren R., Bouwmeester J. (2000) 'De Kansmeter. Een evaluatie ten behœve van de verdere ontwikkeling van het instrument', Den Haag: min SZW.

Decreuse B., Di paola V. (2002), «L'employabilité des chômeurs de longue durée: mise en perspective des littératures théoriques et empiriques. », Revue d'économie politique, $\mathrm{n}^{\mathrm{o}} 2$, pp. 197-227.

De Koning J. (2004), "The reform of the Dutch public employment", Erasmus University, SEOR, Rotterdam, Janvier.

Delarue V. (2005), «Politiques et institutions néerlandaises d'aide au retour à l'emploi», Les Papiers du CERC, $\mathrm{n}^{\circ}$ 2005-03, décembre.
Debauche E., Georges N. (2007), «Construire un outil de profilage des demandeurs d'emploi: défi statistique ou défi politique?», Document de travail du CEE $n^{\circ}$ 91, août.

Delautre G., Georges N. (2007), «De l'utilité d'un outil de profilage des demandeurs d'emploi. Comparaison de trois expériences européennes », L'Essentiel $\mathrm{n}^{\mathrm{0}} 10$, Observatoire de l'ANPE, février.

Eberts R., O’Leary C. (2003), “A New WPRS Profiling Model for Michigan", Upjohn Institute Staff Working Paper no 04-102.

Eberts R., O'Leary R., Wandner S. (2002), Targeting Employment Services, Kalamazoo, MI: W.E. Upjohn Institute for Employment Research.

FARMER B.A. (1993), "Profiling Unemployment Insurance (UI) Claimants", Unemployment insurance program letter $n^{\circ} 45-93$, US Department of Labor.

Fougere D. (2000), «La durée du chômage en France», in J.-P. Fitoussi, O. Passet, J. Freyssinet Réduction $d u$ chômage: les réussites en Europe, rapport du Conseil d'analyse économique $\mathrm{n}^{\circ} 23$, La Documentation française, Paris, pp. 239-259.

FRETS M. (2005), "From chance meter to chance activation, chance exploration, chance observation", $E U$ Profiling Seminar, January 12/14.

Frölish M., LeChNER M., Steiger H. (2004), "Statistically assisted programme selection - International experiences and potential benefits for Switzerland", IFAU working paper, 2004: 1 . 
Georges N. (2006), «Le profilage: outil statistique et/ou mode de coordination?», Document de travail du CEE $\mathrm{n}^{\mathrm{o}} 72$, novembre.

Georges N. (2007), «Profiler les chômeurs?», Connaissance de l'emploi $\mathrm{n}^{\circ} 44, \mathrm{CEE}$, juillet.

GibBins C. (1997), Early Identification Pilots : Results at 12 months, Employment Service, REB113, August.

Glynstrup, J.D., Rosholm, M. (2005), “Employability profiling toolbox", EU Profiling Seminar, January $12 / 14$.

Hammer B. Rosholm M., Svarer M. (2004), “A Danish Profiling System", Institute for the Study of Labor, IZA Discussion Paper n ${ }^{\circ}$ 1418, November.

Hasluck, C. (2004) Targeting Services in the Individual Customer Strategy: The Role of Profiling A Review of Research Evidence, Report for Jobcentre Plus.

Hasluck C. (2006), "Profiling and targeting public employment services: the international experience", $E U$ Profiling Seminar, October 11/13.

Herbillon J.-M. (2004), «Le profiling des demandeurs d'emploi aux Pays-Bas», Travail et Emploi, no 99, juillet.

Hipple S. (1997), "Worker Displacement in an Expanding Economy", Monthly Labor Review, décembre, pp. 2639.

Koning P., VAn Leuvensteijn M. (2000), "Duration dependance in employment insurance and social assistance: consequences of profiling for the unemployed", CPB Research Memorandum $n^{\circ} 163$, Den Haag: CPB.

Lipp R. (2005), "Job Seeker Profiling, The Australian Experience", EU Profiling Seminar, January 12/14.

ObBen J. (2001), “Towards a formal profiling model to foster active labour market policies in New Zealand", Massey University discussion paper.

Ocde (1993), Perspectives de l'emploi, chapitre 3 «Le chômage de longue durée: quelques causes et remèdes $»$.
OCDE (2000), Le service public de l'emploi aux ÉtatsUnis, OCDE, Paris.

Ocde (2002), Perspectives de l'emploi, chapitre 4 «Le chômage de longue durée: tenants et aboutissants».

OCDE (2006), "Activation strategies and performance of employment services in the Netherlands, Germany and the United Kingdom", OECD Report for Joint SZW/ OECD Seminar on Activation Strategies and Performance of Employment Services, The Hague, March 29.

Ocde (2007), Perspectives de l'emploi, Paris, annexe statistique.

O’Leary C. (2006), "Profiling for Public Workforce Investment Programs in the US", EU Profiling Seminar, October $11 / 13$.

Olsen R., Decker P. (2001), “Testing Different Methods of Estimating the Impacts of Worker Profiling and Reemployment Services System", Mimeo June. Washington, DC: Mathematica Policy Research, Inc.

Payne C., Payne J. (2000), "Early Identification of the Long-Term Unemployed", PSI Research Discussion Paper $n^{\circ} 4$.

Pls Ramboll Management (2001), Study on Early Identification of High Risk Unemployed, Final report.

SEN A. (1997), "L'inégalité, le chômage et l'Europe aujourd'hui», Revue internationale du travail, volume $136, \mathrm{n}^{\circ} 2$.

TNO-ARBEID (2003), "Voorspellende waarde van fasering", TNO-bœkrapport 25324, Hoofddorp: TNO.

US Department of Labor (1994), The Worker Profiling and Reemployment Services System: Legislation, Implementation and Research Findings. Washington, D.C.: US Department of Labor, UI Occasional Paper no 94-4.

Wells B. (1998), "Early Identification/Profiling in the United Kingdom" in Early Identification of Jobseekers at Risk of Long-term Unemployment, The Role of Profiling, OCDE. 